Eur Child Adolesc Psychiatry. 2016 January ; 25(1): 67-80. doi:10.1007/s00787-015-0703-7.

\title{
Mechanisms in the relation between GABRA2 and adolescent externalizing problems
}

\author{
Frances L. Wang ${ }^{1}$, Laurie Chassin ${ }^{1}$, Christian Geiser ${ }^{2}$, and Kathryn Lemery-Chalfant ${ }^{1}$ \\ ${ }^{1}$ Department of Psychology, Arizona State University, 950 S. McAllister Ave, P.O. Box 871104, \\ Tempe, AZ 85287-1104, USA \\ 2 Department of Psychology, Utah State University, 2810 Old Main Hill, Logan, UT 84322-2810, \\ USA
}

\begin{abstract}
Conduct problems, alcohol problems and hyperactive-inattentive symptoms co-occur at a high rate and are heritable in adolescence. The $\gamma$-aminobutyric acid A receptor, a 2 gene (GABRA2) is associated with a broad spectrum of externalizing problems and disinhibitory-related traits. The current study tested whether two important forms of disinhibition in adolescence, impulsivity and sensation seeking, mediated the effects of GABRA2 on hyperactive-inattentive symptoms, conduct problems, and alcohol problems. Participants were assessed at two waves (11-17 and 12-18 years old; $N=292$ ). Analyses used the GABRA2 SNP, rs279858, which tags the two complementary (yin-yang) GABRA2 haplotypes. Multiple informants reported on adolescents' impulsivity and sensation seeking and adolescents self-reported their hyperactive-inattentive symptoms, conduct problems and lifetime alcohol problems. Impulsivity mediated the effect of GABRA2 on alcohol problems, hyperactive-inattentive symptoms, and conduct problems, whereas sensation seeking mediated the effect of GABRA2 on alcohol problems (AA/AG geno-types conferred risk). $G A B R A 2$ directly predicted adolescent alcohol problems, but the GG genotype conferred risk. Results suggest that there may be multiple pathways of risk from GABRA2 to adolescent externalizing problems, and suggest important avenues for future research.
\end{abstract}

\section{Keywords}

Conduct problems; Hyperactive-inattentive symptoms; Alcohol problems; GABRA2; Impulsivity; Sensation seeking

\section{Introduction}

Conduct problems, alcohol problems, and hyperactive-inattentive symptoms display moderate-to-high heritability in adolescence and co-occur at a high rate [1, 2]; this cooccurrence may be due in large part to shared genetic factors [1,2]. Although identifying genes that contribute to this shared genetic influence is important, it is also important to

Frances L. Wang franceswang3@gmail.com.

Conflict of interest

The authors declare that they have no conflict of interest. 
identify mechanisms through which shared genes influence these phenotypes. The $\gamma$ aminobutyric acid (GABA) A receptor, a 2 gene (GABRA2), located on chromosome 4, may play a role in externalizing phenotypes. From a biological perspective, there are several factors that might account for this association. GABA acts as the vertebrate brain's main inhibitory neurotransmitter [3], and $\mathrm{GABA}_{\mathrm{A}}$ receptors undergo allosteric modulation by drugs such as ethanol, benzodiazepines, and barbituates [4]. GABAergic interneurons within the ventral tegmental area are also primarily responsible for the inhibitory regulation of dopamine neurons [5, 6], which have long been implicated in impulsive and addictive behaviors [7].

Along these lines, previous studies have shown associations between GABRA2 variation and adolescent externalizing symptoms [8,9], adolescent conduct disorder [10, 11], and adolescent rule-breaking behaviors [12]. However, one recent study did not replicate associations between GABRA2 and adolescent conduct disorder [13]. Although adult studies are lacking, one study found an association between GABRA2 and adult antisocial personality disorder [14]. Thus, the literature largely suggests that GABRA2 relates to conduct problems and externalizing symptoms in adolescent samples and in one adult sample. In contrast, there is little evidence that $G A B R A 2$ directly predicts adolescent alcohol phenotypes [8,11-13]. However, there is more evidence in adult studies [15-24] and one recent meta-analysis [25] that found associations between GABRA2 variants and adult substance use phenotypes. It is possible that $G A B R A 2$ directly predicts adult, but not adolescent, alcohol phenotypes because environmental influences (e.g., peers and family) are more important than genetic influences in predicting adolescent substance use [26]. By adulthood, the impact of genetic influences increases substantially [26], possibly accounting for the more robust prediction of adult substance use by GABRA2.

Interestingly, although Trucco et al. [12] did not find a direct effect of GABRA2 on adolescent alcohol problems, they did find that GABRA2 predicted adolescent alcohol problems indirectly, through mid-adolescent rule-breaking behaviors. Perhaps researchers have not found a direct association between GABRA2 and adolescent alcohol phenotypes because this relation is indirect, and operates through other risk factors. Thus, it is important to examine earlier mediating mechanisms of the relation between GABRA2 and adolescent alcohol problems. Finally, few studies to our knowledge have examined the association between $G A B R A 2$ and hyperactive-inattentive symptoms, which is surprising given that GABRA2 has been previously theorized to confer risk for a broad spectrum of externalizing problems [27].

If GABRA2 does confer risk for a broad spectrum of externalizing behaviors, it would be important to understand the mechanism(s) underlying these associations. Previous research suggests that behavioral disinhibition may be the heritable intermediate phenotype shared among externalizing problems [2] and, therefore, perhaps GABRA2 predicts externalizing problems through behavioral disinhibition. Complicating this proposition, however, are empirical observations that disinhibition is heterogeneous and consists of distinct facets [30]. To account for this heterogeneity, some studies examined associations between GABRA2 and distinct facets of disinhibition, finding that GABRA2 is related to adults' NEO impulsivity (under negative affectivity) [23, 24], and to adolescents' sensation seeking but 
not impulsivity [9]. One study also found that NEO impulsiveness partially mediated the effect of GABRA2 on adults' lifetime alcohol problems [24].

Taken together, previous research suggests that GABRA2 relates to some facets of disinhibition and these facets at least partially mediate the effect of GABRA2 on externalizing problems. However, no studies to our knowledge have examined which facets of disinhibition mediate the relation between GABRA2 and adolescent conduct problems, alcohol problems, and hyperactive-inattentive symptoms.

Understanding whether GABRA2 predicts these externalizing problems indirectly, through disinhibition, will inform the mechanisms that underlie these relations and provide a further test of whether GABRA2 does confer risk for broad externalizing symptomatology. In addition, examining these mediational links is important because a recent meta-analysis demonstrated that genetic effects were stronger on trait measures of impulsivity (e.g., Eysenck Personality Inventory) compared to manifestations of impulsivity in clinical symptoms of attention deficit/hyperactivity disorder [31]. Thus, genetic effects might be more clearly detected when using trait measures of behavioral disinhibition than measures of symptoms of disorder.

Two particularly important facets of disinhibition in adolescence are sensation seeking and impulsivity, which have been differentiated based on neurobiological and developmental evidence [32]. Sensation seeking is an earlier indicator of a socioemotionally driven reward system defined as the tendency to seek out novel/varied experiences and take risks, while impulsivity is an indicator of a deficit in a later developing cognitive control system defined as lack of planfulness and self-control [32]. These two types of dis-inhibition were also found in Whiteside and Lynam's [33] model of impulsivity, corresponding with sensation seeking (a tendency to seek out exciting and novel situations) and lack of premeditation (an inability to carefully think and plan).

Research demonstrates that impulsivity and sensation seeking relate differentially to adolescent externalizing problems. Generally, studies show that impulsivity is a predictor of hyperactive-inattentive symptoms (indeed, impulsivity is a core symptom of attention deficit/hyperactivity disorder) [34-36], conduct problems, and substance use, over and above sensation seeking [35-39]. On the other hand, sensation seeking appears to have more specificity in predicting externalizing problems. Over and above impulsivity, sensation seeking does not uniquely predict hyperactive-inattentive symptoms [35-37], has relatively weak or no associations with conduct problems [34, 35, 38-40], but remains uniquely associated with adolescent and adult substance use [35, 37, 39, 40].

Researchers posited that perhaps impulsivity more broadly predicts all three externalizing problems because those who are impulsive have general deficits in regulating/inhibiting their impulses, thus resulting in the expression of a wide array of problematic behaviors [40]. On the other hand, sensation seeking might specifically predict adolescent substance use because individuals with high sensation seeking have increased motivation for experiencing stimulation and positive emotions [41], and the use of substances might serve to regulate such affective states [40]. Although possibly less robust, the observed relation 
between sensation seeking and conduct problems might be explained by the fact that individuals with conduct problems are more likely to experience low autonomic arousal [42]. Because this state might be perceived as unpleasant, those with conduct problems might regulate this state by seeking stimulating experiences like delinquency [43]. Given their role in adolescent problem behaviors, impulsivity and sensation seeking are important facets of disinhibition to test as mediators in the relation between GABRA2 and externalizing problems.

Of note, the literature on $G A B R A 2$ has largely focused on single nucleotide polymorphisms (SNPs) within two complementary (yin-yang) GABRA2 haplotypes that account for most of the haplotype diversity in Caucasians and Asians [4]. Major, or more frequent, alleles of SNPs within this haplotype block tag the major haplotype and vice versa in these populations. There is some inconsistency regarding which is the risk allele/haplotype from these yin-yang haplotypes and SNPs within them. For example, for the well-studied polymorphism, rs279858 (see "Methods" for more detail), some studies found that the major (A) allele predicted risk for externalizing-related problems [15, 20, 28]. However, others found that the minor $(\mathrm{G})$ allele predicted risk for externalizing phenotypes such as conduct and alcohol problems in predominantly Caucasian samples [12, 16, 23, 24, 29]. Similar inconsistent findings have been observed for other SNPs in the haplotype block and externalizing disorders [17, 19, 21]. Interestingly, one study showed that alcoholics with high trait anxiety had a higher abundance of the major haplotype, while alcoholics with low trait anxiety had a higher abundance of the minor haplotype [69], suggesting that minor vs. major haplotypes of GABRA2 might confer risk for externalizing problems through different mechanisms [4]. More research is needed to understand this phenomenon, especially in adolescence.

Based on this literature, the major aim of the current study was to examine associations between $G A B R A 2$ and adolescent externalizing problems and to test whether impulsivity and/or sensation seeking mediated these relations. These associations were tested in a longitudinal high-risk sample, in which approximately half of the adolescents had parents with substance use disorders. Impulsivity, sensation seeking, baseline conduct problems, alcohol problems, and hyperactive-inattentive symptoms were measured 18 months prior to the outcomes of conduct problems, alcohol problems, and hyperactive-inattentive symptoms. We first tested proposed mediational models without controlling for baseline externalizing problems to simply evaluate associations between the constructs. Next, we tested these models while controlling for baseline externalizing problems to establish the directionality of the relation between impulsivity/sensation seeking and externalizing problems and to test whether GABRA2 predicts change in these externalizing problems over 18 months. Both these models were tested for several reasons. First, because stability in problem behaviors might be more genetically influenced [44] while change in symptoms over time might be more environmentally influenced [45], we wanted to ensure that we were not weakening genetic effects by predicting change in symptoms. Second, researchers have also emphasized the importance of evaluating genetic influences on changes in behavior over time, especially during the critical developmental period of adolescence [28]. 
We hypothesized that GABRA2 would predict sensation seeking, but not impulsivity, based on results reported by Dick et al. [9]. We also expected that sensation seeking would predict alcohol problems and perhaps conduct problems but not hyperactive-inattentive symptoms, and that impulsivity would predict all three phenotypes. Thus, we expected that GABRA2 would predict alcohol problems and perhaps conduct problems indirectly through sensation seeking and that GABRA2 would not directly predict hyper-active-inattentive symptoms nor indirectly predict hyperactive-inattentive symptoms through impulsivity or sensation seeking.

In testing these questions, it is important to acknowledge that a limitation of the candidate gene literature is the lack of replicability across studies [70]. This lack of replicability raises concerns that results from candidate gene studies are false positives, especially those found using small sample sizes such as in the current study $(N=292)$. We reduced the likelihood that our results were false-positive findings in several ways. First, we minimized the number of statistical tests performed by using only one SNP within one gene based on theoretically and biologically plausible reasoning. Second, this particular SNP and gene have shown associations with impulsive personality traits and externalizing problems in numerous other studies and in one meta-analysis [8-25]. In this way, our study serves as a replication of these prior studies. If our findings replicate those from previous studies, this will increase confidence that our results are not due to chance. Third, we included covariates to ensure that associations among $G A B R A 2$, disinhibition, and externalizing problems were not due to associations with other related (i.e., third) variables. Finally, we corrected for multiple testing to further reduce the likelihood of spurious findings.

\section{Methods}

\section{Participants}

Participants were drawn from the third generation of a longitudinal study of familial alcoholism (see Chassin et al. [26] for details). Data were drawn from the sixth wave (referred to as T1) and a follow-up assessment occurring 18 months after the sixth wave (referred to as T2). Adolescents and their parents participated at T1 and only adolescents participated at T2. We included participants who were between 11-17 years old at T1 and 12-18 at T2, had complete genetic data, and were of non-Hispanic Caucasian or Hispanic ethnicity. These inclusion criteria were chosen to capture adolescence, retain as many participants as possible, and reduce heterogeneity in ethnicity. Some adolescents reported fewer lifetime alcohol problems at $\mathrm{T} 2$ than at $\mathrm{T} 1(N=20)$, which is a common phenomenon in adolescent substance use research called recanting [47]. Because reasons for recanting are varied (e.g., problems with recall, social desirability), we excluded those cases, resulting in a final sample of $N=292$. Compared to those excluded, those included were significantly younger at T2, had significantly higher ancestry scores (higher levels of Hispanic ancestry), fewer T1 lifetime alcohol problems, and fewer conduct problems (see Table 1). The lower alcohol and conduct problems of those included are likely due to the fact that they are younger than those excluded, which was purposeful. 


\section{Recruitment and procedure}

In the larger study, families with at least one alcoholic parent (from the first-generation) were recruited using court DUI records, health maintenance organization wellness questionnaires, and community telephone surveys. Telephone surveys were used to recruit families without parental alcoholism, who were matched to families with parental alcoholism on demographic characteristics. See Chassin et al. [46] for details. For the current analyses, at T1, participants (from the third-generation) and their parents (from the second-generation) were interviewed at home. Out-of-state participants were interviewed via telephone or mailed surveys. Buccal and saliva samples using Oragene kits were used to collect biological samples containing DNA. At T2, participants were interviewed via telephone or mailed questionnaires. Participants and parents provided written informed consent and/or assent at all assessments.

\section{Measures}

Demographics-Adolescents self-reported their gender, age, and ethnicity. See Table 1.

Genotyping and SNP selection-Extraction of DNA, standardization and plating were completed in the Department of Psychiatry at the Washington University School of Medicine and genotyping through the Washington University Genome Sequencing Center. 1536 SNPs were designed for genotyping using the Illumina Golden Gate technology that draws on a previous collaboration illustrated in Hodgkinson et al. [48] with substitutions reflecting advances in the literature. Quality control analyses included examining cluster plots to rule out ambiguous genotype calls, checking for Mendelian inconsistencies, incorrect gender assignments and sample swaps, cryptic relatedness, flagging SNPs with low call rates $(<95 \%)$, and deviations from Hardy-Weinberg equilibrium $\left(p<10^{-6}\right)$. We selected rs279858, one of the most widely studied GABRA2 SNPs, based on associations with adolescent externalizing problems [8, 9], adolescent sensation seeking [9], adolescent conduct problems [12], adult alcohol phenotypes [16, 17, 20, 23, 24, 28, 29], and adult drug phenotypes [15]. A recent meta-analysis also found that this SNP was associated with adult alcohol use disorders [25]. Numerous SNPs in high LD $\left(r^{2} \geq 0.80\right)$ with rs 279858 in nonHispanic Caucasian and Hispanic populations (calculated using AMR and EUR 1000 Genomes Phase 1 populations in HaploReg v2; [49]) have been associated with adolescent conduct disorder [10,11], and adult substance use disorders [17, 19, 50]. Thus, rs279858 is a reasonable proxy for these GABRA2 SNPs in both ethnic groups in the current study. This SNP also tags the commonly studied yin-yang haplotypes. Our use of a single, well-studied SNP within GABRA2 that tags other risk variants in GABRA2 is similar to methods of several other studies $[10-12,50]$.

This SNP (rs279858) encodes a silent polymorphism in exon 5 and consists of alleles G (minor) and A (major). In our sample, the minor allele frequency was $37.8 \%$ in nonHispanic Caucasians and $42.3 \%$ in Hispanics. Similar to previous work, we combined nonHispanic Caucasian and Hispanic participants in analyses because both groups had the same minor allele and similar minor allele frequencies [13]. Ancestry informative markers controlled for population stratification (see below). Based on a previous study using a similar sample in terms of age, ethnicity, and high risk for substance use disorders, we coded 
the SNP such that those with the AA and AG genotypes were coded 0 and the GG genotype coded $1[10]$. Genotype did not differ by ancestry (see Table 2).

Adolescent ancestry-Thirty-seven SNPs in the dataset are ancestry informative markers that distinguished between non-Hispanic Caucasian and Hispanic ancestry in previous studies [51]. A principal components analysis using these SNPs indicated that the first component explained $18.99 \%$ of the variance (eigenvalue $=7.03$ ), the second explained $3.36 \%$ (eigen-value $=1.24$ ), and the third explained $3.11 \%$ (eigen-value $=1.02)$. We used the 32 SNPs with loadings greater than 0.30 on the first principal component as indicators of a one-factor model using maximum likelihood estimation. The model fits the data well: $\chi^{2}$ $(464)=824.99, p<0.001$, RMSEA $=0.03, \mathrm{CFI}=0.94, \mathrm{SRMR}=0.03$. Factor scores were saved and used as a covariate and were highly correlated with self-reported ethnicity ( $r=$ $0.86, p<0.001$ ), confirming that these markers differentiate between non-Hispanic Caucasian and Hispanic ancestry in our data. Higher factor scores indicate higher levels of Hispanic ancestry. See Table 1 for descriptive statistics.

Family history density of substance use disorders (FHD)_At T1, adolescents' FHD of alcohol and drug use disorder were calculated separately using methods of Zucker et al. [52] and Stoltenberg et al. [53]. The Computerized Diagnostic Interview Schedule [54] and family member report on the Family History-Research Diagnostic Criteria [55] determined lifetime alcohol and drug disorder (either DSM-III-R or DSM-IV) of adolescents' grandparents and parents. To minimize missingness, data were required from 1 parent and 2 grandparents. Grandparents' lifetime alcohol or drug disorder was weighted by 0.25 , parents' by 0.5 , and relatives without substance disorder by 0 . Summed scores were rescaled from 0 to 2 and averaged to form an FHD composite, which was a covariate (see Table 1).

Item overlap-Because impulsivity is a core symptom of hyperactive-inattentive symptoms, their items might overlap. Impulsivity and sensation seeking both tap the higherorder construct of 'disinhibition' and so their items might overlap as well. One item overlapped between the impulsivity and sensation seeking scales ("do things on the spur of the moment"), and between the impulsivity and hyperactive-inattentive symptoms scales (“do and say things without stopping to think") and were removed.

Adolescent impulsivity and sensation seeking-At T1, mothers, fathers and adolescents reported ( $1=$ strongly disagree, $5=$ strongly agree $)$ on adolescents' impulsivity using the 23-item Junior Eysenck Impulsiveness Questionnaire [56]. Items included, "I often buy things on impulse," and "I often get involved in things I later wish I could get out of." At T1, mothers, fathers, and adolescents reported $(1=$ strongly disagree, $5=$ strongly agree $)$ on adolescents' sensation seeking using six items adapted from Zuckerman's Sensation Seeking Scale [57]. Items included, "I like wild parties," and "I like to have new and exciting experiences, even if they are a little unconventional." For adolescent-, mother- and father-reported impulsivity, $a^{\prime} \mathrm{s}=0.86,0.91$, and 0.91 . For adolescent-, mother- and fatherreported sensation seeking, $a^{\prime} \mathrm{s}=0.63,0.76$, and 0.61. See Table 1 for descriptive data. To reduce the data, a two-factor model of impulsivity and sensation seeking was estimated 
using maximum likelihood estimation with robust standard errors (MLR) and full information maximum likelihood (FIML) to estimate missing data. Mother-, father-, and adolescent-reported impulsivity loaded on an impulsivity factor and mother-, father-, and adolescent-reported sensation seeking on a sensation seeking factor. Within-reporter error covariances were estimated (e.g., error covariance between mother-reported impulsivity and sensation seeking, and so on). This model fit the data well: $\chi^{2}(5)=2.12, p 0.83$; RMSEA = $0.00 ;$ CFI $=1.00 ;$ SRMR 0.01. Standardized factor loadings were significant $(p<=0.001)$ and ranged from 0.52 to 0.71 for impulsivity and from 0.30 to 0.68 for sensation seeking. Within-reporter error covariances were significant $(p<0.001)$ and ranged from 0.43 to 0.55 . The factors were correlated $(r=0.55, p<0.001)$.

Adolescent hyperactive-inattentive symptoms and conduct problems-At T1 and $\mathrm{T} 2$, adolescents reported $(0=$ not true, $1=$ somewhat true, $2=$ very true or often true) on their hyperactive-inattentive symptoms and conduct problems using the DSM-oriented attention deficit/hyperactivity disorder and conduct problems scales of the youth self report [58]. For T1 hyperactive-inattentive symptoms and conduct problems, $\alpha$ 's $=0.83$ and 0.83 . For T2 hyperactive-inattentive symptoms and conduct problems, $\alpha^{\prime} \mathrm{s}=0.80$ and 0.77 . Note that these measures did not include substance use items. Adolescent reports of T1 symptomatology were predictors of corresponding $\mathrm{T} 2$ outcomes for the prospective prediction. Adolescents' reports were used because they were the only informants who reported on symptoms at two waves, allowing us to test change in symptoms. See Table 1 for descriptive statistics.

Adolescent lifetime alcohol problems-At T1 and T2, adolescents self-reported $(0=$ no; $1=$ yes) the lifetime occurrence of alcohol consequences and dependence symptoms. We examined adolescent alcohol problems because research shows that genetic influences are stronger, and environmental influences weaker, on adolescents' problematic alcohol use than on alcohol initiation [59]. Adolescents' reports were used because parents are less accurate reporters of adolescent alcohol use [60] and because adolescents were the only informants who reported on symptoms at two waves. Eighteen consequences as a result of alcohol use (e.g., "did you miss school or work," "did you have problems with family or friends") and 21 alcohol dependence symptoms (e.g., "spent so much time arranging to get alcohol or having them on your mind so much that you had little time for anything else") were summed at each wave to create separate $\mathrm{T} 1$ and $\mathrm{T} 2$ lifetime alcohol problem variables. $\mathrm{T} 1$ alcohol problems was a covariate predicting $\mathrm{T} 2$ alcohol problems for the prospective prediction. See Table 1 for descriptives.

\section{Data analysis}

Analyses used MPlus v.7.2 [61]. Because adolescents' T2 alcohol problems was a count outcome, we compared preliminary models with different distributional assumptions including zero-inflated poisson and zero-inflated negative binomial. Comparison of relative fit indices (AIC, BIC, and $-2 \log$ likelihood) supported modeling T2 alcohol problems using zero-inflated poisson (monte-carlo integration) and, therefore, global fit indices were unavailable. Structural equation modeling tested hypothesized relations using MLR and FIML to estimate missing data on endogenous variables. We estimated paths from GABRA2 
and covariates (ancestry, gender, age, and FHD) to mediators (impulsivity and sensation seeking factors) and T2 outcomes (hyperactive-inattentive symptoms, conduct problems, and alcohol problems). Paths were estimated from mediators to outcomes. Models were estimated with and without controlling for T1 symptoms. $95 \%$ asymmetric confidence limits tested mediation [62]. The type $=$ complex function was used to adjust SEs because participants were nested in families. To correct for multiple testing, we calculated false discovery rate-corrected $p$ values ( $q$ values) using the QVALUE software [71]. Analyses suggested that our sample was adequately powered (0.80) to detect small effect sizes $\left(f^{2}\right.$ 0.027), which are typical in candidate gene research.

\section{Results}

\section{Zero-order correlations}

The AA/AG genotypes were marginally or significantly correlated with higher T1 adolescent- and mother-reported sensation seeking and mother-reported impulsivity. GABRA2 did not correlate with ancestry or other variables. FHD was correlated with all but four disinhibition and/or externalizing variables. Higher impulsivity and sensation seeking were generally correlated with greater externalizing outcomes. Few patterns emerged regarding specificity of relations between disinhibition and externalizing, except fatherreported sensation seeking was only significantly correlated with $\mathrm{T} 1$ conduct problems and $\mathrm{T} 2$ alcohol problems.

\section{Structural equation modeling}

See Table 3 for results (including the $q$ value threshold for each effect) and Fig. 1 for the path model. Results are considered significant if their $q$ values $₫ 0.05$.

Impulsivity and sensation seeking-Those with the AA/AG genotypes had higher sensation seeking and higher impulsivity than those with the GG genotype. Higher FHD significantly predicted higher impulsivity and sensation seeking. Male gender significantly predicted higher impulsivity. No other covariates had main effects on impulsivity or sensation seeking. Results are identical in models that did and did not control for T1 problems.

Alcohol problems-In the model not controlling for $\mathrm{T} 1$ alcohol problems, those with the GG genotype had greater T2 alcohol problems. Non-Hispanic Caucasian ancestry (marginal), older age, and female gender predicted higher T2 alcohol problems. No other covariates predicted T2 alcohol problems. Higher sensation seeking and higher impulsivity predicted greater T2 alcohol problems. All results were identical in the model that controlled for $\mathrm{T} 1$ alcohol problems (which was also a significant predictor) except that non-Hispanic Caucasian ancestry significantly and female gender marginally significantly predicted $\mathrm{T} 2$ alcohol problems.

Conduct problems-In the model not controlling for T1 conduct problems, older age predicted higher $\mathrm{T} 2$ conduct problems. No other covariates or GABRA2 predicted T2 conduct problems. Higher impulsivity, but not sensation seeking, predicted higher T2 
conduct problems. Results were identical when controlling T1 conduct problems (which was also a significant predictor) except age no longer predicted conduct problems.

Hyperactive-inattentive symptoms-In the model not controlling for T1 hyperactiveinattentive symptoms, non-Hispanic Caucasian ancestry and female gender significantly predicted higher T2 hyperactive-inattentive symptoms. No other covariates or GABRA2 predicted T2 hyperactive-inattentive symptoms. Higher impulsivity, but not sensation seeking, uniquely predicted higher T2 hyperactive-inattentive symptoms. Results were identical when controlling T1 symptoms (which was also a significant predictor).

Mediation-Sensation seeking mediated the relation between GABRA2 genotype and alcohol problems both with and without controlling for $\mathrm{T} 1$ alcohol problems $\left(\mathrm{CI}_{95}-1.30\right.$, -0.04 C $\left._{95}-1.26,-0.07\right)$. Impulsivity mediated the relations between $G A B R A 2$ and alcohol problems both with and without controlling for $\mathrm{T} 1$ alcohol problems $\left(\mathrm{CI}_{95}-0.90,0.003\right.$; $\left.\mathrm{CI}_{95}-0.99,-0.07\right)$, conduct problems both with and without controlling for T1 conduct problems ( $\left.\mathrm{CI}_{95}-0.62,-0.04 ; \mathrm{CI}_{95}-1.07,-0.14\right)$, and hyperactive-inattentive symptoms both with and without controlling T1 hyperactive-inattentive symptoms $\left(\mathrm{CI}_{95}-0.11,-0.01\right.$; $\left.\mathrm{CI}_{95}-0.17,-0.03\right)$.

\section{Discussion}

We sought to examine whether impulsivity and/or sensation seeking mediated the relations between GABRA2 genotype and adolescent alcohol problems, conduct problems, and hyperactive-inattentive symptoms. To accomplish this goal, we used a multiple reporter methodology and a high-risk sample in which these relations might be more clearly illuminated due to greater variability in study constructs.

We found that those with the AA or AG genotypes of the GABRA2 SNP rs279858 had higher impulsivity and sensation seeking compared to those with the GG geno-type, even after correcting for multiple testing. This finding partially replicates results from a previous study that found that GABRA2 predicted adolescent sensation seeking [9], providing some confidence in this finding. However, our results differ from this study because they did not find a relation between GABRA2 and adolescent impulsivity. This difference might be due to the fact that we utilized multiple informants' reports on the Eysenck Impulsiveness Questionnaire to characterize our measure of impulsivity while they utilized adolescent selfreports on the Barratt Impulsivity scale. Genetic association tests will likely be more reliable when using multiple reporters, rather than only self-reports, of adolescents' disinhibition due to reductions in informant bias that subsequently serve to better capture the trait of interest. Interestingly, previous studies [23, 24] found a relation between GABRA2 and adults' NEOImpulsiveness, which reflects impulsivity under negative affectivity. Nonetheless, because few studies have shown an association between GABRA2 variation and impulsive behavior characterized specifically by a lack of planfulness and cognitive control, this association should be considered as preliminary until replicated in other studies.

However, our results and those of previous studies collectively suggest that GABRA2 is implicated in several distinct facets of disinhibition. Taken together, perhaps GABRA2 
variation predisposes adolescents to broad disinhibition, rather than to one specific facet. In addition, previous research suggests that common genetic influences underlie the association between impulsivity and sensation seeking [63]. Based on our results and those of previous studies, it is possible that this GABRA2 polymorphism or other polymorphisms in high LD might contribute to this common genetic basis.

We found that impulsivity uniquely predicted levels of hyperactive-inattentive symptoms, conduct problems and alcohol problems, as well as change in these symptoms over 18 months. On the other hand, we found that sensation seeking uniquely predicted only alcohol problems and change in these symptoms over 18 months. All of these effects survived correction for multiple testing. These findings are consistent with previous studies [34-40]. Results confirm the notion that impulsivity confers broad risk for multiple externalizing problems, possibly because general deficits in impulse control and inhibition result in the expression of a wide array of problematic behaviors [40]. Results also confirm the relative specificity of sensation seeking in predicting alcohol problems. This unique relation may be due to the fact that individuals with high sensation seeking have an increased drive for reward and stimulation, which they attempt to satisfy by using/misusing alcohol [40, 41]. It is this alcohol misuse that likely leads to the development of alcohol problems. Finally, although some studies found evidence for a unique, albeit weak, effect of sensation seeking on conduct problems $[35,36,38,39]$, the lack of such an association in our study might reflect the fact that impulsivity is simply a more salient pathway to conduct problems than sensation seeking. It is impressive that impulsivity and sensation seeking predicted change in externalizing problems over 18 months given the high stability in externalizing problems across $\mathrm{T} 1$ and $\mathrm{T} 2$. This suggests that impulsivity and sensation seeking precede these problem behaviors, consistent with the vulnerability, or predisposition model of personality/ temperament [64]. This model posits that personality traits represent a predisposition to experience certain problem behaviors.

We found that disinhibition mediated the relation between GABRA2 genotype and adolescent alcohol problems. More specifically, we found that the AA/AG geno-types conferred greater risk for adolescent sensation seeking and impulsivity, which both in turn predicted adolescent-reported alcohol problems as well as change in these symptoms over an 18-month period. These results are similar to another study using an adult sample which found that NEO impulsiveness mediated the relation between GABRA2 and alcohol problems [24]. Taken together, it is possible that disinhibitory personality traits mediate the relation between GABRA2 and alcohol problems. However, based on the previous literature and current findings, it remains unclear exactly which aspect(s) of disinhibition mediate this relation.

Similarly, the AA/AG genotypes also predicted adolescent-reported conduct problems and hyperactive-inattentive symptoms (as well as their change over 18 months) indirectly through adolescent impulsivity. Our finding partially replicates previous research which found a link between GABRA2 and conduct problems [10-12]. However, our results differ from these studies because we only found an indirect, not a direct, link between GABRA2 and conduct problems. Unfortunately, few studies to our knowledge have examined the mechanisms in the relation between GABRA2 and conduct problems and hyperactive- 
inattentive symptoms, or the direct effect of GABRA2 on hyperactive-inattentive symptoms. Thus, these findings must be considered as preliminary until they can be replicated by future studies.

In addition to indirect effects, GABRA2 genotype also had a direct effect on adolescent alcohol problems that survived correction for multiple testing. Interestingly, this effect was such that those with the GG genotype had higher alcohol problems, which is opposite from the indirect effects showing that the AA/AG genotypes predicted externalizing problems through disinhibition. These effects held in models that did and did not control for T1 alcohol problems, suggesting that the switch in risk allele is not due to prediction of change in alcohol problems over 18 months. It is impressive that this GABRA2 SNP predicted change in alcohol problems over 18 months, especially given the high correlation between T1 and T2 alcohol problems. As stated earlier, the risk allele for rs279858 (as well as for other SNPs in the same haplotype block) has been inconsistent in the literature. Some studies found that the major (A) allele was associated with increased risk for externalizing phenotypes [15, 20,28], while other studies including a meta-analysis found that the minor (G) allele was associated with increased risk for externalizing phenotypes [12, 16, 23, 25, 29].

Our results suggest that one possibility for the mixed findings is that GABRA2 may have more than one pathway to externalizing outcomes, which has been previously proposed and empirically observed by other researchers using adult samples [4, 69]. Using our results as an example, one pathway might be through the influence of the $\mathrm{A} /$ major alleles on disinhibition. Although less clear, multiple mechanism(s) might be involved in other pathways of risk. For instance, one study found that presence of the $\mathrm{G}$ allele of rs 279858 predicted adolescent substance use through rule-breaking behaviors [12]. Interestingly, numerous studies reported associations between GABRA2 variation (including rs279858) and increased positive subjective alcohol effects [65-67], possibly because $\mathrm{GABA}_{\mathrm{A}}$ receptors undergo allosteric modulation by ethanol, benzodiazepines and barbituates [4]. Thus, perhaps the GG genotype increases risk for adolescent alcohol problems through its effect on positive subjective responses to alcohol in our sample. This is in line with our findings because we only found a direct effect of the GG genotype on adolescent alcohol problems. Note, however, that the GABRA2 risk alleles were inconsistent in the prior studies on positive subjective response as well. Therefore, these are merely possibilities and other mechanisms might be responsible, which might be the topic of future research. The present results are consistent with previous theories proposing that GABRA2 confers risk for a spectrum of externalizing problems [27], and indicate that this risk might operate through more than one mechanism.

Moreover, it is important to note that if different alleles of GABRA2 do predict externalizing phenotypes through different mechanisms, the fact that many of these mechanisms might also be correlated could confound the direction of association. For example, if opposing risk alleles within $G A B R A 2$ actually predict different, but correlated, intermediate phenotypes, then the effect of GABRA2 on one of the intermediate phenotypes might be masked or reversed if the other phenotype is not included in the model. To further understand the role of GABRA2 on externalizing problems, it will be important to elucidate these possible 
intermediate phenotypes and test them simultaneously and/or use these intermediate phenotypes as covariates. Based on this reasoning, it should be emphasized that the current results are not necessarily informative as to which phenotypes are related to major vs. minor alleles within GABRA2 SNPs. There may be other intermediate phenotypes not tested in the current study that act as confounding variables. Thus, although our results provide very preliminary insights into the mediating mechanisms in the relation between GABRA2 and externalizing phenotypes, they also point to the importance of examining other possible mediators as alternatives. Our results inform directions for future research and provide additional evidence that $G A B R A 2$ risk alleles might differently predict externalizing problems across studies because multiple mechanisms of risk exist $[4,69]$.

Interestingly, GABRA2 did not have direct effects on hyperactive-inattentive symptoms or conduct problems in the final model, nor did GABRA2 have significant zero-order correlations with alcohol problems, hyperactive-inattentive symptoms, or conduct problems. On the other hand, GABRA2 variation directly predicted the latent factors of impulsivity and sensation seeking in the final model and demonstrated several zero-order correlations with manifest impulsivity and sensation seeking variables. Because GABRA2 variation was more consistently associated with disinhibition than with externalizing symptoms, this suggests that it is more strongly related to the personality-related underpinnings of externalizing problems than with the symptoms themselves (which are likely more heterogeneous). This is in line with a recent meta-analysis that showed larger genetic effects on trait compared to diagnostic phenotypes [31]. Results demonstrate the utility of including phenotypes that might be more proximal to genetic influences to elucidate the influence of genes on clinical outcomes [72]. It is possible that non-replications in the literature might be due to the failure to consider possible intermediate phenotypes. Of note, in the final model females had greater adolescent hyperactive-inattentive symptoms. This stands in contrast to the majority of studies showing that males have greater hyperactive-inattentive symptoms than females [68]. However, female gender predicts the unique variance in hyperactive-inattentive symptoms after partialling out impulsivity and sensation seeking, possibly explaining the unusual direction of risk. In post hoc analyses that removed impulsivity and sensation seeking (but not other predictors) from the model, gender no longer significantly predicted hyperactive-inattentive symptoms $(\beta=-0.08, \mathrm{~ns})$.

The current study had several methodological strengths. Our use of multiple informants in the factor model of impulsivity and sensation seeking increased confidence in the findings because associations were likely not due to informant bias. We utilized longitudinal data to prospectively predict change in externalizing outcomes over 18 months. This allowed us to establish the directionality of the effect from disinhibition to externalizing outcomes and assess the role of the SNP on behavior change. We also tested these models without controlling for T1 externalizing symptoms to ensure that genetic effects were not weakened by predicting change in externalizing problems, especially because $\mathrm{T} 1$ and $\mathrm{T} 2$ externalizing problems were moderately stable. Our use of a high-risk sample likely more clearly illuminated the pathways of interest due to greater variability in study constructs. In addition, to reduce the possibility of false-positive findings, we tested only theoretically driven associations, examined only one SNP based on biological and theoretical reasoning, 
included covariates, and corrected for multiple testing. Furthermore, this particular SNP has been widely studied and many of our results replicated prior findings.

There were also limitations. We examined single-gene associations, which have small effect sizes. Although the current study had the power to detect small effect sizes, we acknowledge that our sample was relatively small, which increases the chance that significant effects were false-positive findings. Complex phenotypes such as disinhibition and externalizing problems are polygenic. There are many other variants within the GABRA2 gene and across the genome that were not examined in the current study but will likely be important to consider in the future. Although several of our results replicated findings from previous studies, the current study did not have a replication sample. Therefore, this study awaits replication with a larger sample, generalization to different ethnic groups, and should be considered preliminary until replicated in an independent sample.

In summary, the current study replicated previous research which found that GABRA2 variation predicted sensation seeking [9] and alcohol problems [15-25], and that disinhibitory personality traits mediated the association between GABRA2 variation and alcohol problems [24]. Findings novel to the current study include the prediction of impulsivity and the mediating role of impulsivity in the relation between GABRA2 and hyperactive-inattentive symptoms and conduct problems. These findings should be viewed with caution until replicated in future studies. Importantly, our results suggest directions for future research. First, because genes predict change in behavior over time [28], future research might attempt to unpack these developmental processes. Second, it is possible that different risk alleles within GABRA2 might be involved in different pathways of risk for externalizing outcomes $[4,69]$. If this is true, it might help explain the conflicting directions of associations found in the literature among GABRA2 and externalizing problems. It would also suggest the importance of investigating intermediate phenotypes to better understand the role of GABRA2 on externalizing problems. Finally, our results illustrate the value of examining intermediate phenotypes as mediators in the relation between genes and clinical outcomes because these relations cannot be assumed to be direct [72]. In these ways, findings from the current study help to clarify and extend the literature.

\section{Acknowledgments}

This work was supported by the National Institute of Alcohol Abuse and Alcoholism: R01 AA016213 and R21 AA022097-02 to Laurie Chassin, P50 AA11998-01 to the Midwest Alcohol Research Center, and F31 AA023128-01 to Frances Wang. Approval was obtained by the Institutional Review Boards at Arizona State University (IRB\# 0506000017) and Washington University in St. Louis (IRB\# 201110243).

\section{References}

1. Hicks BM, Foster KT, Iacono WG, McGue M. Genetic and environmental influences on the familial transmission of externalizing disorders in adoptive and twin offspring. JAMA Psychiatr. 2013; 70:1076-1083.

2. Young SE, Stallings MC, Corley RP, Krauter KS, Hewitt JK. Genetic and environmental influences on behavioral disinhibition. Am J Med Genet B Neuropsychiatr Genet. 2000; 96:684-695.

3. Barnard EA, Skolnick P, Olsen RW, et al. Subtypes of $\gamma$-aminobutyric acid receptors: $^{-}$ classification on the basis of subunit structure and receptor function. Pharmacol Rev. 1998; 50:291313. [PubMed: 9647870] 
4. Enoch MA. The role of $\mathrm{GABA}_{\mathrm{A}}$ receptors in the development of alcoholism. Pharmacol Biochem Be. 2008; 90:95-104.

5. Johnson SW, North RA. Opioids excite dopamine neurons by hyperpolarization of local interneurons. J Neurosci. 1992; 12:483-488. [PubMed: 1346804]

6. Steffensen SC, Svingos AL, Pickel VM, Henriksen SJ. Electrophysiological characterization of GABAergic neurons in the ventral tegmental area. J Neurosci. 1998; 18:8003-8015. [PubMed: 9742167]

7. Comings DE, Blum K. Reward deficiency syndrome: genetic aspects of behavioral disorders. Prog Brain Res. 2000; 126:325-341. [PubMed: 11105655]

8. Dick DM, Latendresse SJ, Lansford JE, et al. Role of GABRA2 in trajectories of externalizing behavior across development and evidence of moderation by parental monitoring. Arch Gen Psychiatr. 2009; 66:649-657. [PubMed: 19487630]

9. Dick D, Aliev F, Latendresse S, et al. How phenotype and developmental stage affect the genes we find: GABRA2 and impulsivity. Twin Res Hum Genet. 2013; 16:661-669. [PubMed: 23561058]

10. Dick DM, Bierut L, Hinrichs A, et al. The role of GABRA2 in risk for conduct disorder and alcohol and drug dependence across developmental stages. Behav Genet. 2006; 36:577-590. [PubMed: 16557364]

11. Sakai JT, Stallings MC, Crowley TJ, et al. Test of association between GABRA2 (SNP rs279871) and adolescent conduct/ alcohol use disorders utilizing a sample of clinic referred youth with serious substance and conduct problems, controls and available first degree relatives. Drug Alcohol Depend. 2010; 106:199-203. [PubMed: 19783384]

12. Trucco EM, Villafuerte S, Heitzeg MM, Burmeister M, Zucker RA. Rule breaking mediates the developmental association between GABRA2 and adolescent substance abuse. J Child Psychol Psyc. 2014 doi:10.1111/jcpp.12244.

13. Melroy WE, Stephens SH, Sakai JT, et al. Examination of genetic variation in GABRA2 with conduct disorder and alcohol abuse and dependence in a longitudinal study. Behav Genet. 2014; 44:356-367. [PubMed: 24687270]

14. Dick DM, Agrawal A, Schuckit M, et al. Marital status, alcohol dependence, and GABRA2: evidence for gene-environment correlation and interaction. J Stud Alcohol Drugs. 2006; 67:185194.

15. Agrawal A, Edenberg HJ, Foroud T, et al. Association of GABRA2 with drug dependence in the collaborative study of the genetics of alcoholism sample. Behav Genet. 2006; 36:640-650. [PubMed: 16622805]

16. Covault J, Gelernter J, Hesselbrock V, Nellissery M, Kranzler HR. Allelic and haplotypic association of GABRA2 with alcohol dependence. Am J Med Genet B Neuropsychiatr Genet. 2004; 129:104-109. [PubMed: 15274050]

17. Edenberg HJ, Dick DM, Xuei X, et al. Variations in GABRA2, encoding the alpha 2 subunit of the GABA(A) receptor, are associated with alcohol dependence and with brain oscillations. Am J Hum Genet. 2004; 74:705-714. [PubMed: 15024690]

18. Fehr C, Sander T, Tadic A, Lenzen KP, Anghelescu I, Klawe C. Confirmation of association of the GABRA2 gene with alcohol dependence by subtype-specific analysis. Psychiatr Genet. 2006; 16:9-17. [PubMed: 16395124]

19. Lappalainen J, Krupitsky E, Remizov M, et al. Association between alcoholism and gamma-amino butyric acid alpha2 receptor subtype in a Russian population. Alcohol Clin Exp Res. 2005; 29:493-498. [PubMed: 15834213]

20. Lind P, MacGregor S, Agrawal A, Heath AC, Martin NG, Whit-field JB. The role of GABRA2 in alcohol dependence, smoking and illicit drug use in an Australian population sample. Alcohol Clin Exp Res. 2008; 32:1721-1731. [PubMed: 18727688]

21. Philibert RA, Gunter TD, Beach SRH, et al. Role of GABRA2 on risk for alcohol, nicotine, and cannabis dependence in the Iowa adoption studies. Psychiatr Genet. 2009; 19:91-98. [PubMed: 19672139]

22. Soyka M, Preuss UW, Hesselbrock V, Zill P, Koller G, Bondy B. GABA-A2 receptor subunit gene (GABRA2) polymorphisms and risk for alcohol dependence. J Psychiatr Res. 2008; 42:184-191. [PubMed: 17207817] 
23. Villafuerte S, Heitzeg MM, Foley S, et al. Impulsiveness and insula activation during reward anticipation are associated with genetic variants in GABRA2 in a family sample enriched for alcoholism. Mol Psychiatry. 2012; 17:511-519. [PubMed: 21483437]

24. Villafuerte S, Strumba V, Stoltenberg SF, Zucker RA, Bur-meister. Impulsiveness mediates the association between GABRA2 SNPs and lifetime alcohol problems. Genes Brain Behav. 2013; 12:525-531. [PubMed: 23566244]

25. Li D, Sulovari A, Cheng C, Zhao H, Kranzler HR, Gelernter J. Association of $\gamma$-aminobutyric acid A receptor a2 gene (GABRA2) with alcohol use disorder. Neuropsychopharmacology. 2014; 39:907-918. [PubMed: 24136292]

26. Kendler KS, Schmitt E, Aggen SH, Prescott CA. Genetic and environmental influences on alcohol, caffeine, cannabis, and nicotine use from early adolescence to middle adulthood. Arch Gen Psychiatry. 2008; 65:674-682. [PubMed: 18519825]

27. Dick DM. Identification of genes influencing a spectrum of externalizing psychopathology. Curr Dir Psychol Sci. 2007; 16:331-335.

28. Dick DM, Cho SB, Latendresse SJ, et al. Genetic influences on alcohol use across stages of development: GABRA2 and longitudinal trajectories of drunkenness from adolescence to young adulthood. Addict Biol. 2013 doi:10.1111/adb.12066.

29. Bauer LO, Covault J, Harel O, et al. Variation in GABRA2 predicts drinking behavior in project MATCH subjects. Alcohol Clin Exp Res. 2007; 31:1780-1787. [PubMed: 17949392]

30. Dick DM, Smith G, Olausson P, et al. Review: understanding the construct of impulsivity and its relationship to alcohol use disorders. Addict Biol. 2010; 15:217-226. [PubMed: 20148781]

31. Jonas KG, Markon KE. A meta-analytic evaluation of the endophenotype hypothesis: effects of measurement paradigm in the psychiatric genetics of impulsivity. J Abnorm Psychol. 2014; 123:660-675. [PubMed: 24978691]

32. Steinberg L. A dual systems model of adolescent risk-taking. Dev Psychobiol. 2010; 52:216-224. [PubMed: 20213754]

33. Whiteside SP, Lynam DR. The five factor model and impulsivity: using a structural model of personality to understand impulsivity. Pers Individ Dif. 2001; 30:669-689.

34. Handley ED, Chassin L, Haller MM, Bountress KE, Dandreaux D, Beltran I. Do executive and reactive disinhibition mediate the effects of familial substance use disorders on adolescent externalizing outcomes? J Abnorm Psychol. 2011; 120:528-542. [PubMed: 21668077]

35. Miller J, Flory K, Lynam D, Leukefeld C. A test of the four-factor model of impulsivity-related traits. Personal Individ Differ. 2003; 34:1403-1418.

36. Miller DJ, Derefinko KJ, Lynam DR, Milich R, Fillmore MT. Impulsivity and attention deficithyperactivity disorder: subtype classification using the UPPS impulsive behavior scale. J Psychopathol Behav Assess. 2010; 32:323-332. [PubMed: 21765593]

37. Bates ME, Labouvie EW. Personality environment constellations and alcohol use: a processoriented study of intraindividual change during adolescence. Psychol Addict Behav. 1995; 9:2325.

38. Derefinko K, DeWall CN, Metze AV, Walsh EC, Lynam DR. Do different facets of impulsivity predict different types of aggression? Aggress Behav. 2011; 37:223-233. [PubMed: 21259270]

39. Lynam DR, Miller JD. Personality pathways to impulsive behavior and their relations to deviance: results from three samples. J Quant Criminol. 2004; 20:319-341.

40. Castellanos-Ryan N, Rubia K, Conrod P. Sensation seeking and common and unique variance in conduct disorder and substance misuse. Alcohol Clin Exp Res. 2011; 35:1-15. [PubMed: 20958327]

41. Comeau N, Stewart SH, Loba P. The relations of trait anxiety, anxiety sensitivity, and sensation seeking to adolescents' motivations for alcohol, cigarette, and marijuana use. Addict Behav. 2001; 26:803-825. [PubMed: 11768546]

42. Raine A. Biosocial studies of antisocial and violent behavior in children and adults: a review. $\mathrm{J}$ Abnorm Child Psychol. 2002; 30:311-326. [PubMed: 12108763]

43. Frick PJ, Morris AS. Temperament and developmental pathways to conduct problems. J Clin Child Adolesc Psychol. 2004; 33:54-68. [PubMed: 15028541] 
44. O'Connor TG, Neiderhiser JM, Reiss D, Hetherington EM, Plomin R. Genetic contributions to continuity, change, and co-occurrence of antisocial and depressive symptoms in adolescence. $\mathrm{J}$ Child Psychol Psyc. 1998; 39:323-336.

45. McGue M, Bacon S, Lykken DT. Personality stability and change in early adulthood: a behavioral genetic analysis. Dev Psychol. 1993; 29:96-109.

46. Chassin L, Barrera M, Bech K, Kossak-Fuller J. Recruiting a community sample of adolescent children of alcoholics: a comparison of three subject sources. J Stud Alcohol. 1992; 53:316-319. [PubMed: 1619925]

47. Fendrich M, Yun Soo Kim J. Multiwave analysis of retest artifact in the national longitudinal survey of youth drug use. Drug Alcohol Depend. 2001; 62:239-253. [PubMed: 11295329]

48. Hodgkinson CA, et al. Addictions biology: haplotype-based analysis for 130 candidate genes on a single array. Alcohol Alcohol. 2008; 43:505-515. [PubMed: 18477577]

49. Ward LD, Kellis M. HaploReg: a resource for exploring chromatin states, conservation, and regulatory motif alterations within sets of genetically linked variants. Nucleic Acids Res. 2012; 40:D930-D934. [PubMed: 22064851]

50. Perry BL, Pescosolido BA, Bucholz K. Gender-specific gene-environment interaction in alcohol dependence: the impact of daily life events and GABRA2. Behav Genet. 2013; 43:402-414. [PubMed: 23974430]

51. Tian C, Gregersen PK, Seldin MF. Accounting for ancestry: population substructure and genomewide association studies. Hum Mol Genet. 2008; 17:R143-R150. [PubMed: 18852203]

52. Zucker RA, Ellis DA, Fitzgerald HE. Developmental evidence for at least two alcoholisms. I. biopsychosocial variation among pathways into symptomatic difficulty. Ann NY Acad Sci. 1994; 708:134-146. [PubMed: 8154674]

53. Stoltenberg SF, Mudd SA, Blow FC, Hill EM. Evaluating measures of family history of alcoholism: density versus dichotomy. Addiction. 1998; 93:1511-1520. [PubMed: 9926555]

54. Robins, LN.; Cottler, LB.; Bucholz, KK.; Compton, WM.; North, CS.; Rourke, KM. Diagnostic interview schedule for the DSMIV (DIS-IV). Washington University School of Medicine; MO: 2000.

55. Endicott, J.; Andreasen, N.; Spitzer, RL. Family history-research diagnostic criteria. Biometrics Research. New York State Psychiatric Institute; New York: 1975.

56. Eysenck SBG, Easting G, Pearson PR. Age norms for impulsiveness, venturesomeness and empathy in children. Pers Indiv Differ. 1985; 5:315-321.

57. Zuckerman, M. Sensation seeking: beyond the optimal level of arousal. Lawrence Erlbaum Associates; Hillsdale: 1979.

58. Achenbach, TM.; Rescorla, LA. Manual for the ASEBA School-Age Forms \& Profiles. University of Vermont, Research Center for Children, Youth, \& Families; Burlington, VT: 2001.

59. Rhee SH, Hewitt JK, Young SE, Corley RP, Crowley TJ, Stallings MC. Genetic and environmental influences on substance initiation, use, and problem use in adolescents. Arch Gen Psychiatry. 2003; 60:1256-1264. [PubMed: 14662558]

60. Fisher SL, Bucholz KK, Reich W, Fox L, Kuperman S, Kramer J. Teenagers are right-parents do not know much: an analysis of adolescent-parent agreement on reports of adolescent substance use, abuse, and dependence. Alcohol Clin Exp Res. 2006; 30:1699-1710. [PubMed: 17010137]

61. Muthén, LK.; Muthén, BO. Mplus User's Guide. Seventh Edition.. Los Angeles, CA: 1998-2012.

62. MacKinnon DP, Lockwood CM, Williams J. Confidence limits for the indirect effect: distribution of the product and resampling methods. Multivariate Behav Res. 2004; 39:99-128. [PubMed: 20157642]

63. Hur Y, Bouchard TJ. The genetic correlation between impulsivity and sensation seeking traits. Behav Genet. 1997; 27:455-463. [PubMed: 9336082]

64. Rothbart, MK.; Bates, JE. Temperament.. In: Damon, W.; Eisenberg, N., editors. Handbook of child psychology, vol 3., Social, emotional, personality development. Wiley; New York: 2006. p. 88-166.

65. Haughey HM, Ray LA, Finan P, Villanueva R, Niculescu M, Hutchison KE. The human GABA(A) receptor alpha2 gene moderates the acute effects of alcohol and brain mRNA expression. Genes Brain Behav. 2008; 7:447-454. [PubMed: 18005236] 
66. Pierucci-Lagha A, Covault J, Feinn R, et al. GABRA2 alleles moderate the subjective effects of alcohol, which are attenuated by finasteride. Neuropsychopharmacology. 2005; 30:1193-1203. [PubMed: 15702134]

67. Uhart M, Weerts EM, Mccaul ME, et al. GABRA2 markers moderate the subjective effects of alcohol. Addict Biol. 2013; 18:357-369. [PubMed: 22501025]

68. Cohen P, Cohen J, Kasen S, et al. An epidemiological study of disorders in late childhood and adolescence, 1: age- and gender-specific prevalence. J Child Psychol Psychiatr. 1993; 34:851-867.

69. Enoch MA, Schwartz L, Albaugh B, Virkkunen M, Goldman D. Dimensional anxiety mediates linkage of GABRA2 haplotypes with alcoholism. Am J Med Genet B Neuropsychtr Genet. 2006; 141:599-607.

70. Vassos E, Collier DA, Fazel S. Systematic meta-analyses and field synopsis of genetic association studies of violence and aggression. Mol Psychiatry. 2014; 19:471-477. [PubMed: 23546171]

71. Storey JD. A direct approach to false discovery rates. J R Stat Soc Series B Stat Methodol. 2002; 64:479-498.

72. Gottesman II, Gould TD. The endophenotype concept in psychiatry: etymology and strategic intentions. Am J Psychiatry. 2003; 160:636-645. [PubMed: 12668349] 


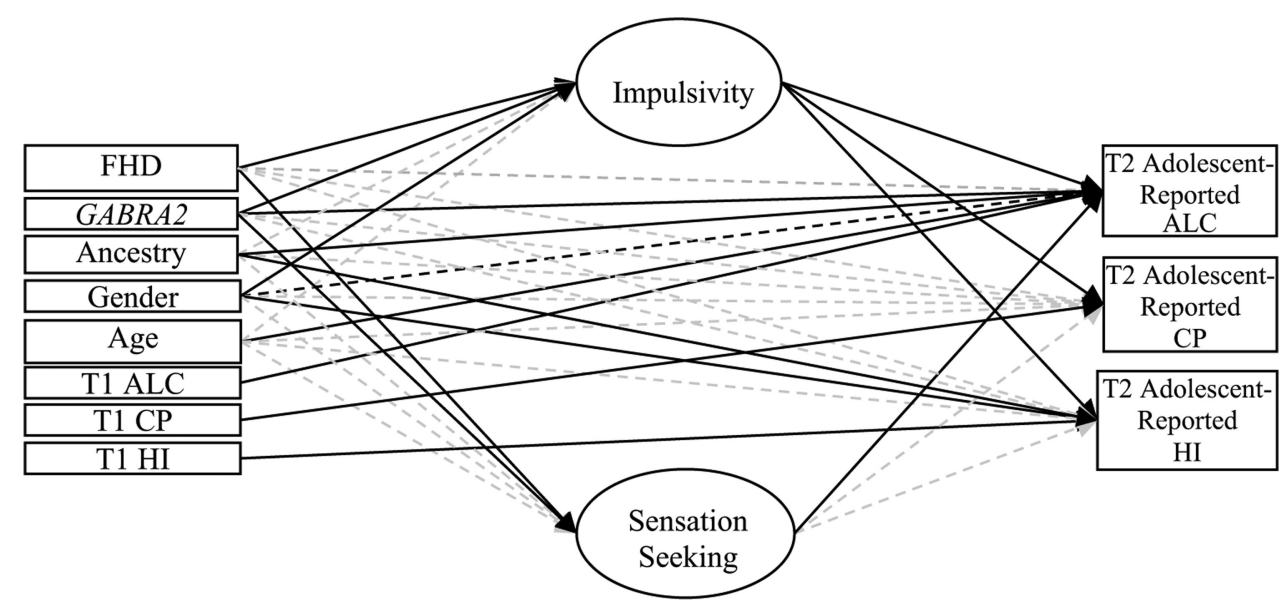

Fig. 1.

Final prospective path model. The model controlling for T1 symptoms is shown for simplicity. Correlations between exogenous variables, a correlation between impulsivity and sensation seeking, and correlations between outcome residual variables are not shown for ease of presentation. Solid black lines refer to statistically significant paths $(q \leq 0.05)$, dashed black lines refer to marginally significant paths $(q 0.10)$, and grayed dashed lines refer to nonsignificant paths $(q>\leq 0.10)$. FHD family history density of substance use disorders, $T 1$ time 1, $T 2$ time $2, H I$ hyperactive-inattentive symptoms, $C P$ conduct problems, $A L C$ alcohol problems. $N=292$ 
Table 1

Descriptive statistics, $t$ tests, and Chi-square analyses comparing included vs. excluded participants

\begin{tabular}{lllc}
\hline Variables & Included $\boldsymbol{M}$ (SD) & Excluded $\boldsymbol{M}$ (SD) & $\boldsymbol{t}$ \\
\hline Ancestry marker factor score & $0.06(0.91)$ & $-0.10(0.92)$ & $-2.11^{*}$ \\
T1 age $^{a}$ & $12.66(1.72)$ & $12.88(5.47)$ & 0.7 \\
T2 age $^{b}$ & $13.97(1.81)$ & $14.43(3.39)$ & $2.0{ }^{*}$ \\
Family history density of substance use disorder & $0.44(0.41)$ & $0.44(0.41)$ & -0.11 \\
Impulsivity (adolescent) & $2.88(0.62)$ & $2.98(0.64)$ & 1.84 \\
Sensation seeking (adolescent) & $3.15(0.73)$ & $3.20(0.79)$ & 0.77 \\
Impulsivity (mother) & $2.64(0.73)$ & $2.69(0.74)$ & 0.89 \\
Sensation seeking (mother) & $2.99(0.74)$ & $3.00(0.77)$ & 0.14 \\
Impulsivity (father) & $2.67(0.71)$ & $2.76(0.64)$ & 1.49 \\
Sensation seeking (father) & $3.03(0.63)$ & $3.07(0.63)$ & 0.79 \\
T1 alcohol problems (adolescent) & $0.05(0.42)$ & $0.33(1.18)$ & 3.99 \\
T1 hyperactivity-impulsivity (adolescent) & $3.26(2.91)$ & $3.10(2.68)$ & -0.73 \\
T1 conduct problems (adolescent) & $1.98(2.71)$ & $2.33(3.02)$ & 1.49 \\
T2 alcohol problems (adolescent) & $0.18(0.68)$ & $0.28(1.38)$ & 1.12 \\
T2 hyperactivity-impulsivity (adolescent) & $2.76(2.59)$ & $2.63(2.48)$ & -0.64 \\
T2 conduct problems (adolescent) & $1.86(2.61)$ & $1.70(2.81)$ & -0.66 \\
\hline
\end{tabular}

\begin{tabular}{|c|c|c|c|}
\hline & $\%$ & $\%$ & $\chi^{2}$ \\
\hline Biological parent alcohol disorder ${ }^{c}$ & $44.3 \%$ biological parent alcohol disorder & $42.6 \%$ biological parent alcohol disorder & - \\
\hline Biological parent drug disorder ${ }^{d}$ & $40.5 \%$ biological parent drug disorder & $34.7 \%$ biological parent drug disorder & - \\
\hline \multirow[t]{3}{*}{ Ethnicity } & $71.1 \%$ non-Hispanic Caucasian & $47 \%$ non-Hispanic Caucasian & - \\
\hline & $28.9 \%$ Hispanic & $30.9 \%$ Hispanic & \\
\hline & & $22.1 \%$ Other & \\
\hline \multirow[t]{2}{*}{$G A B R A 2$} & $79.7 \% \mathrm{AA} / \mathrm{AG}$ & $81.8 \% \mathrm{AA} / \mathrm{AG}$ & 0.37 \\
\hline & $20.3 \% \mathrm{GG}$ & $18.2 \% \mathrm{GG}$ & \\
\hline Gender & $48.1 \%$ female & $45.9 \%$ female & 0.3 \\
\hline
\end{tabular}

$N=292$

Parentheses designate the reporter

$T$ tests and $\chi^{2}$ analyses were not performed for non-study variables (e.g., self-reported ethnicity, biological parent alcohol and drug use disorder)

$*$

$p \leq 0.05$

$a_{\text {Time } 1}$

$b_{\text {Time } 2}$

${ }^{c}$ At least one biological parent was diagnosed with alcohol use disorder according to DSM-IV criteria

${ }^{d}$ At least one biological parent was diagnosed with drug use disorder according to DSM-IV criteria

Eur Child Adolesc Psychiatry. Author manuscript; available in PMC 2017 January 01. 

Table 3

Standardized path coefficients and standard errors for final path models

\begin{tabular}{|c|c|c|c|c|c|}
\hline \multirow[t]{2}{*}{ Predictors } & \multicolumn{2}{|l|}{ Mediators } & \multicolumn{3}{|l|}{ Outcomes } \\
\hline & T1 impulsivity $\beta(S E)$ & $\begin{array}{l}\text { T1 sensation } \\
\text { seeking } \beta(S E)\end{array}$ & $\begin{array}{l}\text { T2 alcohol } \\
\text { problems } \beta(S E)\end{array}$ & $\begin{array}{l}\text { T2 conduct } \\
\text { problems } \beta(S E)\end{array}$ & $\begin{array}{l}\text { T2 hyperactive- } \\
\text { inattentive } \\
\text { symptoms } \beta(S E)\end{array}$ \\
\hline \multicolumn{6}{|c|}{ Results without controlling for $\mathrm{T} 1$ symptoms } \\
\hline GABRA2 & $-0.15(0.05)^{*}$ & $-0.21(0.08)^{*}$ & $0.35(0.10)^{* *}$ & $0.10(0.06)$ & $0.03(0.07)$ \\
\hline Ancestry & $0.08(0.08)$ & $0.06(0.09)$ & $-0.15(0.08)^{\dagger}$ & $-0.08(0.06)$ & $-0.16(0.07)^{*}$ \\
\hline Gender & $0.18(0.07)^{*}$ & $0.12(0.07)$ & $-0.31(0.13)^{*}$ & $-0.02(0.06)$ & $-0.21(0.06)^{* *}$ \\
\hline FHD & $0.28(0.06)^{* *}$ & $0.25(0.08)^{*}$ & $-0.06(0.10)$ & $0.03(0.06)$ & $0.06(0.06)$ \\
\hline T1 age & $0.03(0.07)$ & $0.02(0.08)$ & $0.38(0.11)^{*}$ & $0.17(0.06)^{*}$ & $0.09(0.06)$ \\
\hline Impulsivity & - & - & $0.55(0.19)^{*}$ & $0.57(0.11)^{* *}$ & $0.59(0.10)^{* *}$ \\
\hline Sensation seeking & - & - & $0.49(0.19)^{*}$ & $0.01(0.16)$ & $-0.07(0.13)$ \\
\hline \multicolumn{6}{|c|}{ Results while controlling for $\mathrm{T} 1$ symptoms } \\
\hline GABRA2 & $-0.15(0.05)^{*}$ & $-0.21(0.08)^{*}$ & $0.40(0.09)^{* *}$ & $0.06(0.06)$ & $0.02(0.07)$ \\
\hline Ancestry & $0.09(0.08)$ & $0.06(0.09)$ & $-0.17(0.09)^{*}$ & $-0.08(0.06)$ & $-0.16(0.06)^{*}$ \\
\hline Gender & $0.17(0.07)^{*}$ & $0.10(0.07)$ & $-0.23(0.14)^{\dagger}$ & $-0.04(0.06)$ & $-0.14(0.05)^{*}$ \\
\hline FHD & $0.28(0.06)^{* *}$ & $0.24(0.08)^{*}$ & $-0.02(0.10)$ & $0.01(0.06)$ & $0.08(0.06)$ \\
\hline T1 age & $0.01(0.07)$ & $-0.003(0.08)$ & $0.37(0.11)^{*}$ & $0.08(0.05)$ & $0.04(0.05)$ \\
\hline T1 symptoms ${ }^{a}$ & - & - & $0.11(0.04)^{*}$ & $0.46(0.06)^{* *}$ & $0.41(0.06)^{* *}$ \\
\hline Impulsivity & - & - & $0.50(0.24)^{*}$ & $0.31(0.11)^{*}$ & $0.36(0.10)^{* *}$ \\
\hline Sensation seeking & - & - & $0.53(0.23)^{*}$ & $0.04(0.14)$ & $-0.05(0.11)$ \\
\hline
\end{tabular}

False discovery rate-corrected $p$ value (i.e., $q$ value) thresholds are reported

T1 Time 1, T2 Time 2

* $q \leq 0.05$

**

$q \leq 0.001$

${ }^{\dagger} q \leq 0.10, N=292$

${ }^{a}$ Corresponding T1 symptoms (e.g., T1 ALC predicting T2 ALC) 